\section{Tacrolimus ointment is a safe adjuvant treatment for lichen sclerosus in boys}

Lichen sclerosus of the penis can cause scarring at the glans, urethral stricture, and may be associated with squamous cell carcinoma. Topical tacrolimus is effective against lichen sclerosus in female adults, and Ebert et al. have now shown that tacrolimus ointment is a safe adjuvant treatment for lichen sclerosus in boys after circumcision.

Of 222 boys who underwent circumcision at a pediatric urology clinic in Regensburg, Germany, 25 were diagnosed with lichen sclerosus by histopathology. Twenty boys (aged $5-16$ years) entered a phase II trial of $0.1 \%$ tacrolimus ointment. Eleven boys had disease confined to the removed foreskin; the other nine boys had involvement at the glans and/or meatus. All 20 boys were disease free after 21 days of treatment. Median follow-up was 13 months (range 8-28 months). Two relapses occurred-one in each group-after 6 months and 8 months of follow-up; however, both patients were successfully treated by a 3-week course of topical tacrolimus. Four patients reported mild initial itching, which resolved spontaneously. No other adverse events were reported. A further 18 boys from Regensburg with lichenoid symptoms suggestive of early lichen sclerosus were followed up without adjuvant treatment; none developed full-scale disease.

The authors point out that circumcision alone was insufficient to treat disease in $45 \%$ of the patients in their study. They conclude that tacrolimus ointment is a safe, tolerable adjuvant treatment for lichen sclerosus in boys. Placebo-controlled trials are now needed to prove the effectiveness of this treatment.

Original article Ebert AK et al. (2008) Safety and tolerability of adjuvant topical tacrolimus treatment in boys with lichen sclerosus: a prospective phase 2 study. Eur Urol 54: 932-937

\section{Erectile function in end-stage renal disease is not improved by renal transplantation}

Erectile dysfunction (ED) is common in men with end-stage renal disease (ESRD); however, whether normalization of kidney function by renal transplantation can improve ED in such individuals is not known. Mirone et al. used the International Index of Erectile Function (IIEF) to assess ED before and 1 year after successful renal transplantation in 78 men with ESRD.

The overall prevalence of ED at baseline was $87.17 \%$, and was higher in men aged $<45$ years $(n=32)$ than in those aged $\geq 45$ years $(n=46$; $90.62 \%$ vs $82.60 \%)$. One year after transplantation, the overall prevalence of ED had risen to $91.02 \%$. The mean total IIEF score after transplantation was not significantly different from baseline in men aged $\geq 45$ years, but was significantly decreased in those aged $<45$ years $(P=0.01)$. A significant decrease in mean total IIEF score was reported in patients with dyslipidemia $(P=0.008)$ and in those receiving methylprednisolone $(P=0.0204)$ or ciclosporin $(P=0.045)$. Those patients using $\beta$-blockers, $\mathrm{Ca}^{2+}$ antagonists or angiotensin-convertingenzyme inhibitors had a significant decrease in the mean IIEF erectile function domain score compared with baseline values $(P=0.0355$, $P=0.0204$ and $P=0.045$, respectively).

ED is prevalent in men with ESRD, and is not affected by renal transplantation in men aged 45 years or over; however, transplantation is associated with deterioration in erectile function in men younger than 45 years. The authors recommend psychological counseling and careful moderation of medication for men with ESRD and ED undergoing renal transplantation.

Original article Mirone V et al. (2008) Renal transplantation does not improve erectile function in hemodialysed patients. Eur Urol [doi:10.1016/j.eururo.2008.09.020] 